Supporting Information

\title{
Facile Synthesis of 3D NiCoP@ NiCoPO Core-Shell Nanostructures with Boosted Catalytic Activity toward Oxygen Evolution Reaction
}

Mi-Xue Jin, Yu-Lu Pu, Zi-Juan Wang, Zheng Zhang, Lu Zhang, * Ai-Jun Wang, and Jiu-Ju Feng *

Key laboratory of the Ministry of Education for Advanced Catalysis Materials, College of Chemistry and Life Sciences, College of Geography and Environmental Sciences, Zhejiang Normal University, Jinhua 321004, China

*Corresponding author: zhanglu@zjnu.edu.cn (L. Zhang)

jjfeng@zjnu.cn (J.J.Feng) 


\section{Experimental Section}

\section{Materials}

Nickel chloride $\left(\mathrm{NiCl}_{2}\right)$, potassium hexacyanocobaltate(III) $\left(\mathrm{K}_{3} \mathrm{Co}(\mathrm{CN})_{6}\right)$, sodium hypophosphite $\left(\mathrm{NaH}_{2} \mathrm{PO}_{2}\right)$, and sodium borohydride $\left(\mathrm{NaBH}_{4}\right)$ were purchased from Shanghai Aladdin Industrial Corporation (Shanghai, China). Other reagents were of analytical grade and used without further purification. Commercial ruthenium dioxide $\left(\mathrm{RuO}_{2}\right)$ was purchased from STREM Chemical Corporation (Shanghai, China).

\section{Synthesis of 3D core-shelled NiCoP@NiCoPO $\left(\mathrm{CS}-\mathrm{NiCoP} @ \mathrm{NiCoPO}_{\mathbf{x}}\right)$}

Light blue $\mathrm{NiCl}_{2} / \mathrm{K}_{3} \mathrm{Co}(\mathrm{CN})_{6}$ cyanogel was obtained by mixing $1.0 \mathrm{~mL}$ of $1 \mathrm{M} \mathrm{NiCl}_{2}$ and $2.0 \mathrm{~mL}$ of $0.5 \mathrm{M} \mathrm{K} \mathrm{K}_{3} \mathrm{Co}(\mathrm{CN})_{6}$ aqueous solutions at room temperature under ultrasonication. Then, $6.0 \mathrm{~mL}$ of a mixed solution containing $200 \mathrm{mM} \mathrm{NaH} \mathrm{PO}_{2}$ and $475 \mathrm{mM} \mathrm{NaBH} 4$ was added into the as-obtained $\mathrm{NiCl}_{2} / \mathrm{K}_{3} \mathrm{Co}(\mathrm{CN})_{6}$ cyanogel under vigorous stirring, followed by remaining static for $12 \mathrm{~h}$. Finally, the resultant black CS-NiCoP@ NiCoPO mixture were separated by centrifugation at $6000 \mathrm{rpm}$ for 5 min, washed consecutively with water and ethanol, and then dried at $60{ }^{\circ} \mathrm{C}$ in a vacuum oven for $12 \mathrm{~h}$.

To confirm the role of $\mathrm{NaH}_{2} \mathrm{PO}_{2}$, the referenced NiCo product without $\mathrm{P}$ (defined as NiCo-NS for simplicity) were prepared by the $\mathrm{NiCl}_{2} / \mathrm{K}_{3} \mathrm{Co}(\mathrm{CN})_{6}$ cyanogel-reduction system, by using $\mathrm{NaBH}_{4}$ as the single reducing agent. 


\section{Electrochemical measurements}

All electrochemical experiments were carried out on a CHI 660D electrochemical workstation ( $\mathrm{CH}$ Instruments, Shanghai, Chenhua Co.) with a Gamry RDE 710 rotating disk electrode in $\mathrm{O}_{2}$-saturated $1 \mathrm{M} \mathrm{KOH}$ electrolyte at room temperature. A graphite rod auxiliary electrode and a saturated calomel reference electrode (SCE) were used. All of the potentials refer to reversible hydrogen electrode (RHE), where $E_{\mathrm{RHE}}=E_{\mathrm{SCE}}+0.241 \mathrm{~V}+0.0591 \mathrm{pH}$. For preparation of a working electrode, $4 \mathrm{mg}$ of the catalyst was well dispersed in $2 \mathrm{~mL}$ of ethanol (EtOH) containing $10 \mu \mathrm{L}$ of $5 \mathrm{wt} \%$ Nafion solution under strong ultrasonication to obtain an evenly distributed suspension. Then, $18.0 \mu \mathrm{L}$ of the resulting suspension was laid on the surface of a glassy carbon electrode (GCE, $5 \mathrm{~mm}$ in diameter, $0.196 \mathrm{~cm}^{2}$ ). After drying at $40{ }^{\circ} \mathrm{C}$, the working electrode was obtained, and the specific metal loading on the electrode surface was about $0.18 \mathrm{mg} \mathrm{cm}^{-2}$.

\section{Physical characterization}

Scanning electron microscopy (SEM) images were taken on a Hitachi S-4800 scanning electron microscope operating at $5 \mathrm{kV}$. Transmission electron microscopy (TEM) images were obtained with a JEOL JEM-2100F electron microscope operated at $200 \mathrm{kV}$. X-ray diffraction (XRD) data were collected on a Model D/max-rC X-ray diffractometer coupled with a $\mathrm{CuK} \alpha$ radiation source $(1.5406 \AA$ ) at $40 \mathrm{kV}$ and 100 mA. X-ray photoelectron spectroscopy (XPS) experiment was conducted on a Thermo VG Scientific ESCALAB 250 spectrometer with an $\mathrm{Al} \mathrm{K \alpha}$ radiator. The binding 
energy was calibrated with respect to $\mathrm{C} 1 \mathrm{~s}$ at $284.8 \mathrm{eV}$. The compositions of the catalysts were determined from the energy dispersive spectrum (EDS). 

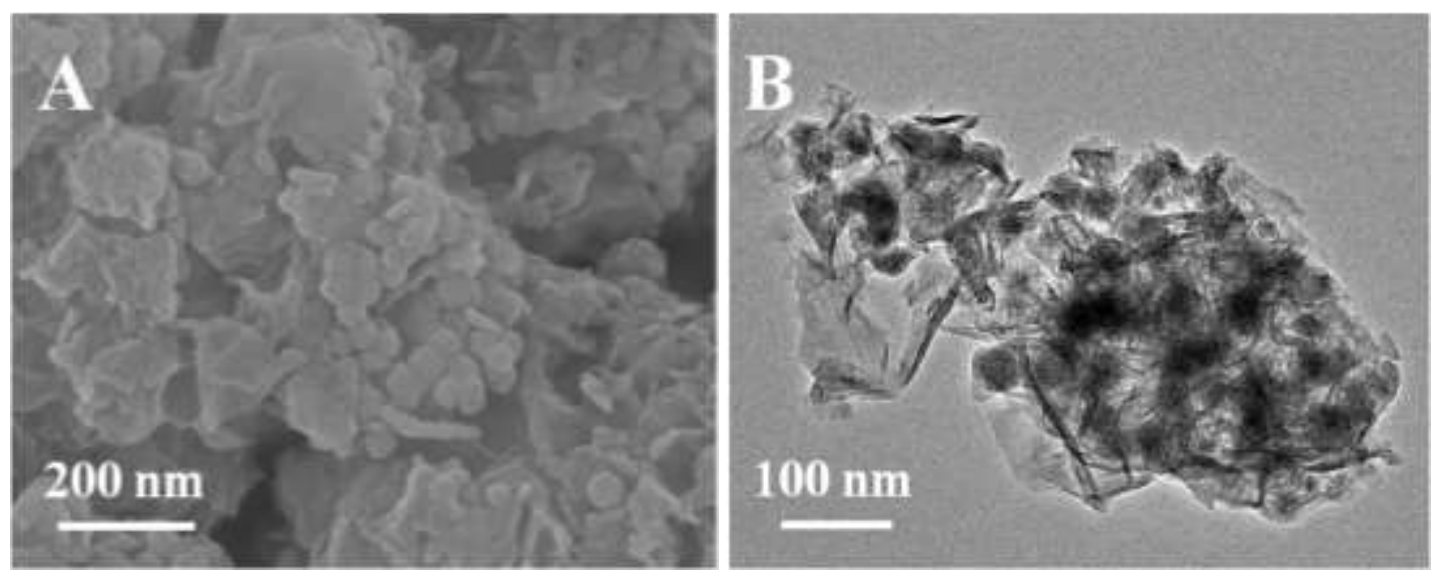

Figure S1. SEM (A) and TEM (B) images of NiCo-NS. 

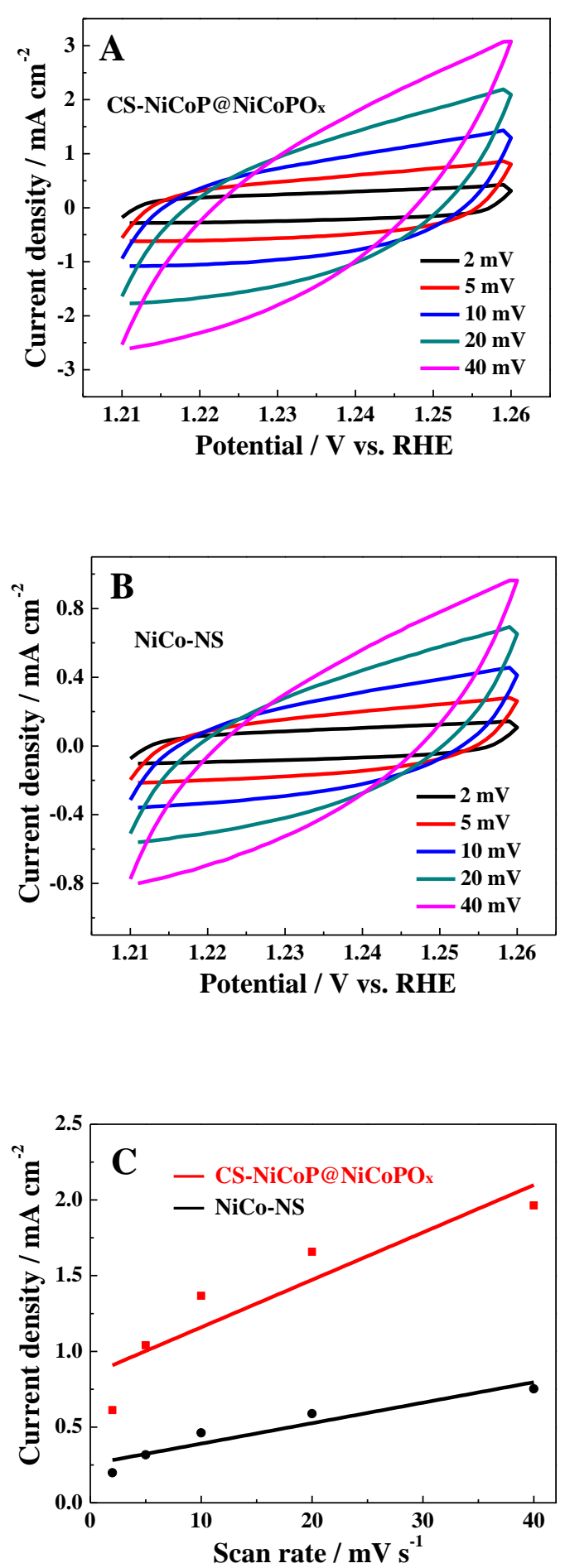

Figure S2. CV curves of the (A) CS-NiCoP@ NiCoPO and (B) NiCo-NS catalysts in $\mathrm{O}_{2}$-saturated $1 \mathrm{M} \mathrm{KOH}$ at different scan rates. (C) Plots of the current density vs. scan rate at $1.24 \mathrm{~V}$. 


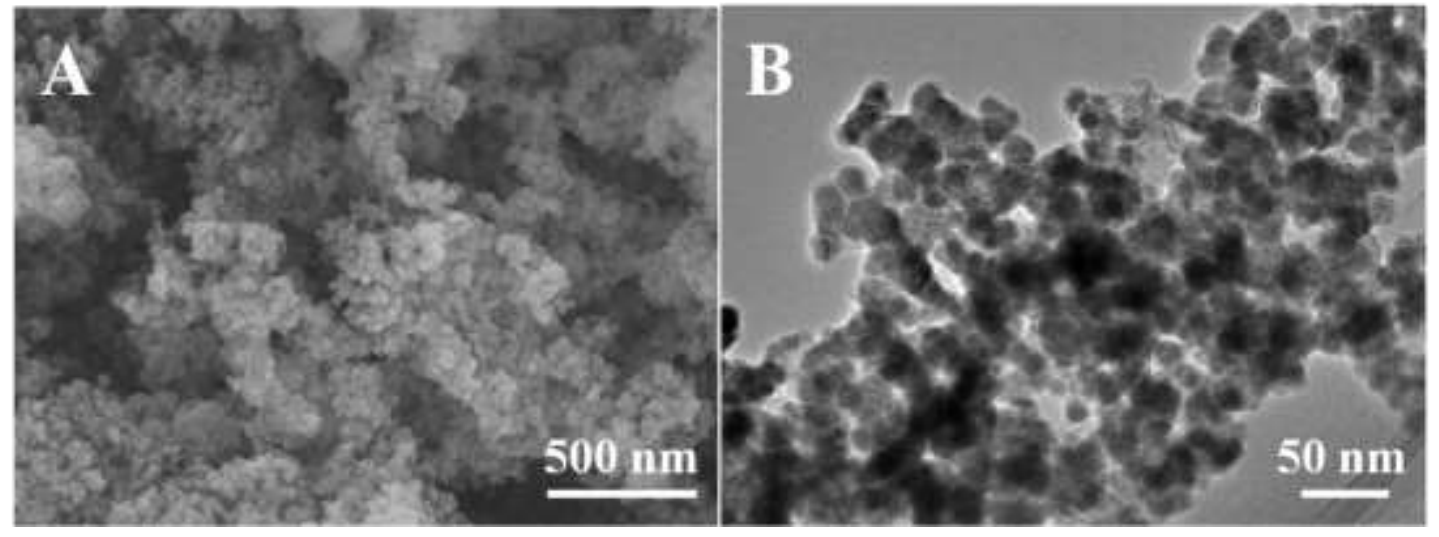

Figure S3. SEM (A) and TEM (B) images of CS-NiCoP@ $\mathrm{NiCoPO}_{x}$ after 5 h OER stability test. 